\title{
Xiang-Qi-Tang Increases Avian Pathogenic Escherichia coli-Induced Survival Rate and Regulates Serum Levels of Tumor Necrosis Factor Alpha, Interleukin-1 and Soluble Endothelial Protein C Receptor in Chicken
}

\author{
Chang-Liang He, ${ }^{a, \#}$ Ben-Dong Fu, ${ }^{a, \#}$ Hai-Qing Shen, ${ }^{a}$ Xiao-Lin JiAng, ${ }^{b}$ Chang-Shuai Zhang, ${ }^{a}$ \\ Shuai-Cheng Wu, ${ }^{a}$ Wei ZHU, ${ }^{c}$ and $\mathrm{Xu}-\mathrm{Bin} \mathrm{WeI}{ }^{*, a}$ \\ ${ }^{a}$ Department of Clinical Veterinary Medicine, College of Animal Science and Veterinary Medicine, Jilin University; Xi'an \\ Road 5333, Changchun, Jilin 130062, P. R. China: ${ }^{b}$ Beijing Key Laboratory of Traditional Chinese Veterinary Medicine, \\ Beijing University of Agriculture; Beijing 102206, P. R. China: and ${ }^{c}$ College of Animal Science, Guizhou University; No. \\ 14 Xiahui Road, Guiyang, Guizhou 550025, P. R. China. \\ Received October 9, 2010; accepted November 7, 2010; published online December 8, 2010
}

\begin{abstract}
Xiang-Qi-Tang (XQT) is a Chinese herbal formula containing Rhizoma Cyperi, Andrographis paniculata and Astragalus membranaceus. The present study investigated the effects of XQT on the mortality and inflammatory mediators in a chicken model challenged with avian pathogenic Escherichia coli (APEC). To detect the effect of XQT, the chickens were pretreated with the formula $12 \mathrm{~h}$ before being challenged with $10^{8}$ colony forming unit (CFU) of APEC. The results showed that $0.6 \mathrm{~g} / \mathrm{kg}$ XQT significantly elevated the survival rate of infected chickens. To further investigate the mechanism of decreasing mortality of XQT, we examined plasma inflammatory mediator levels. The levels of tumor necrosis factor alpha (TNF- $\alpha$ ), interleukin-1 (IL-1) and soluble endothelial protein $C$ receptor (sEPCR) were significantly increased in chickens challenged with APEC alone, whereas chickens pretreated with $0.6 \mathrm{~g} / \mathrm{kg}$ XQT showed marked decrease of these inflammatory mediator levels during the death peak. Taken together, this study demonstrates that XQT has protective effects in APEC-treated chickens. The action mechanisms of XQT involve anti-inflammation and antithrombotic activity. These findings may contribute to future research on the action mechanisms of this formula, as well as prevention of or therapy for avian colibacillosis.
\end{abstract}

Key words Xiang-Qi-Tang; avian pathogenic Escherichia coli; inflammation; thrombosis; chicken

Avian pathogenic Escherichia coli (APEC) causes colibacillosis in chickens, turkeys, and other avian species ${ }^{1)}$ and is responsible for significant economic losses by the poultry industry. APEC induces extraintestinal infections, including respiratory infection (airsacculitis and pneumonia), pericarditis, perihepatitis, and sepsis of poultry. Sepsis is the most prevalent form of colibacillosis, which affects chickens of all ages. ${ }^{2)}$ The pathophysiology of sepsis is extremely complex, being characterized by systemic inflammation, impaired protein $\mathrm{C}$ (PC) pathway, coagulopathy, multiple organ failure, and endothelium injury. ${ }^{3-5}$ ) When APEC or other bacterium release their endotoxin into the bloodstream, the host defense causes a rapid inflammatory response to cope with the following sepsis. Many cytokines, such as tumor necrosis factor alpha (TNF- $\alpha$ ) and interleukin-1 (IL-1), are known to be important soluble mediators responsible for coordinating this response, ${ }^{6}$ ) and even to participate in the pathogenesis of hemodynamic involvement and cellular lesions. ${ }^{7)}$

On the other hand, bacterial endotoxin can change the endothelial lining of blood vessels from an anticoagulant, profibrinolytic surface into one that promotes thrombosis. ${ }^{8)}$ Septic patients frequently manifest disseminated intravascular coagulation producing multiple organ system failure. ${ }^{9)}$ The PC system, mainly consisting of PC, thrombomodulin (TM), and endothelial PC receptor (EPCR), plays a pivotal role in the hemostatic abnormalities of septic patients. ${ }^{10)} \mathrm{Ac}-$ tivated PC (APC), converted from circulating zymogen PC by the thrombin-TM-EPCR complex, has been shown to reduce the rate of death due to severe sepsis. ${ }^{11)}$ It is reported

* To whom correspondence should be addressed. e-mail: weixub@163.com \# These authors contributed equally to this work. that recombinant APC can protect microcirculation in an animal model of neonatal septic shock. ${ }^{12)}$

Xiang-Qi-Tang (XQT), a Chinese herbal formula, is composed of Rhizoma Cyperi, Andrographis paniculata and Astragalus membranaceus with an optimal ratio. Rhizoma Cyperi has been reported to prevent the onset of allergies or improve allergic symptoms. ${ }^{13)}$ It appears that Andrographis paniculata may have a number of potential therapeutic uses, including as an antibacterial ${ }^{14)}$ or antiinflammatory agent and as a treatment for chemically induced hepatotoxicity. ${ }^{15)}$ Astragalus membranaceus also possesses a wide range of pharmacological activities, such as inhibition of inflammation and platelet aggregation, protection against liver failure, and endothelium injury. ${ }^{16-18)}$ These findings suggest that XQT has potential benefits for chickens challenged with APEC. Therefore, in this study, we investigated the effect of XQT on the survival of chickens challenged with APEC and on inflammatory mediators in serum, such as TNF- $\alpha$, IL-1 and soluble endothelial protein $\mathrm{C}$ receptor (sEPCR). According to these, we hoped to provide a theoretical basis for further research on XQT and its application in prevention of or therapy for avian colibacillosis.

\section{MATERIALS AND METHODS}

Main Reagents Rhizoma Cyperi (40 g), Andrographis paniculata $(30 \mathrm{~g})$, Astragalus membranaceus (30 g) (Changchun Hongjian pharmacy products) were soaked in 10 volumes of water for $1 \mathrm{~h}$ in a round-bottom flask. The fluid was heated for $1 \mathrm{~h}$ and then filtered while still hot. The dregs 
of the medical decoction were sequentially extracted with eight and six times the volume of water, respectively, following the same procedure described above. The three filtrates were combined and condensed by decompression to yield the XQT solution with an approximate concentration of $1 \mathrm{~g} / \mathrm{ml}$. The XQT solution was put through a $0.22 \mu \mathrm{m}$ pore filter and stored at $4{ }^{\circ} \mathrm{C}$. Chicken TNF- $\alpha$, IL- 1 and sEPCR enzymelinked immunosorbent assay (ELISA) kits were purchased from Groundwork Biotechnology Diagnosticate Ltd. (Canada).

Animals Used in Infection Models One-day-old white leghorn specific pathogen free (SPF) chickens were obtained from the Experimental Animal Center of Jilin University and raised to $21 \mathrm{~d}$ old in positive-pressure Horsfall-Bauer-type isolation units with food and water ad libitum. All animal experiments were performed in accordance with the guide for the Care and Use of Laboratory Animals published by Jilin University.

Inoculum Preparation E. coli O78 (CVCC1418) was purchased from the China Veterinary Culture Collection Center. The preparation of APEC suspension for infecting chickens was performed as the method described by Antão et al. ${ }^{19)}$ Briefly, one loopful of the stock culture was plated on Luria-Bertani (LB) agar and incubated at $37^{\circ} \mathrm{C}$ for $18 \mathrm{~h}$. A single colony of this fresh culture was inoculated in $5 \mathrm{ml}$ of LB broth and incubated at $37^{\circ} \mathrm{C}$ for $18 \mathrm{~h}$ with agitation. Then, $18 \mathrm{~h}$ cultures were diluted $1: 100$ in fresh LB broth and grown at $37^{\circ} \mathrm{C}$ with agitation to about $2 \times 10^{8}$ colony forming unit $(\mathrm{CFU}) / \mathrm{ml}$. After incubation, cultures were harvested by centrifugation at $5000 \times \boldsymbol{g}$ for $10 \mathrm{~min}$ and the cell pellet was resuspended in phosphate-buffered saline (PBS) to a concentration of $2 \times 10^{9} \mathrm{CFU} / \mathrm{ml}$.

Infection Model and XQT Treatment To determine a suitable dosage of APEC suspension for infection, chickens (20 in each group) were inoculated subcutaneously with a $0.2-\mathrm{ml}$ suspension containing $10^{9}, 10^{8}, 10^{7} \mathrm{CFU}$ of the virulent strain and normal saline as a control. The survival rate was assessed every $12 \mathrm{~h}$ for $5 \mathrm{~d}$. The suitable dosage was defined as that which caused $80-90 \%$ lethality over $5 \mathrm{~d}$ of observation.

To evaluate the effects of XQT on chickens challenged with APEC, chickens were divided into five groups $(n=20$ for each group): APEC group, low dosage group $(0.3 \mathrm{~g} / \mathrm{kg}$ XQT), middle dosage group $(0.6 \mathrm{~g} / \mathrm{kg}$ XQT $)$, high dosage group $(1.2 \mathrm{~g} / \mathrm{kg} \mathrm{XQT)}$ and control group. The chickens were injected subcutaneously with $0.2 \mathrm{ml}$ APEC suspension (containing $10^{8} \mathrm{CFU}$ ) or saline for the control group $12 \mathrm{~h}$ after administration of XQT. Survival in each group was monitored every $12 \mathrm{~h}$ for $6 \mathrm{~d}$.

Detection of Plasma Inflammatory Mediator To further investigate the effects of XQT, the chicken plasma inflammatory mediator was monitored. All chickens were handled following the above procedure. Based on the survival rate of the experiment, we chose to use a dosage of $0.6 \mathrm{~g} / \mathrm{kg}$ $\mathrm{XQT}$ in this experiment. Chickens were inoculated with $10^{8} \mathrm{CFU}$ of APEC $12 \mathrm{~h}$ after pretreatment of XQT. Blood samples were obtained at $12 \mathrm{~h}, 24 \mathrm{~h}, 48 \mathrm{~h}$ and $72 \mathrm{~h}$ by wing vein blood collection. The blood sample was immediately centrifuged, and serum was stored at $-20^{\circ} \mathrm{C}$ until measurement of inflammatory mediator concentrations. Concentrations of TNF- $\alpha$, IL-1 and sEPCR in serum were determined by using an ELISA kit. Concentrations were determined in duplicates of each sample.

Statistical Analysis The data are expressed as mean \pm standard deviation. Differences in the survival of groups were assessed by Fisher's exact test. The inflammatory mediator significance of differences between the APEC control group and the XQT-treated group or the control group were assessed by one-way ANOVA (Dunnett's $t$ test) and Student's $t$ test. Statistical difference was accepted at $p<0.05$.

\section{RESULTS}

Determination of Suitable Inoculated Dosage of APEC The CFU of APEC on survival rates in the chicken model is shown in Fig. 1. After being injected with APEC, the death peak of infected chickens was 24 to $48 \mathrm{~h}$. Chickens inoculated with $10^{9} \mathrm{CFU}$ of APEC all died in $60 \mathrm{~h}$. Chickens challenged with $10^{8} \mathrm{CFU}$ of APEC showed a $20 \%$ survival rate after $5 \mathrm{~d}$, while those receiving $10^{7} \mathrm{CFU}$ of APEC showed a $70 \%$ survival rate. Therefore, $10^{8} \mathrm{CFU}$ of APEC was the suitable dosage in subsequent experiments.

Effects of XQT on the Survival Rate of Chickens Challenged with APEC As shown in Fig. 2, chickens were given different doses of XQT by oral administration and then inoculated with $10^{8} \mathrm{CFU}$ of APEC after $12 \mathrm{~h}$. Chickens in each group began to die about $24 \mathrm{~h}$ after challenge, but the mortality of the APEC group was higher than other XQTtreated groups, indicating that XQT can protect chickens at initial stages of infection. At the end, the survival rates in the XQT pretreated groups did not show a dose-dependent man-

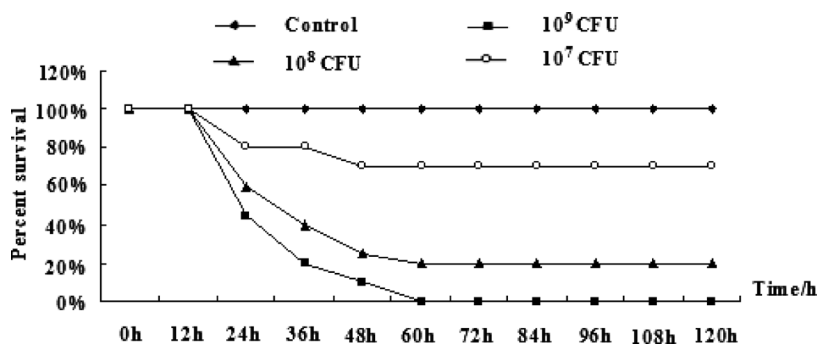

Fig. 1. The Survival Rates of Chickens Challenged with APEC of Different Bacterial Counts

Chickens were inoculated with $10^{9}, 10^{8}$ or $10^{7} \mathrm{CFU}$ of APEC by subcutaneous injection without treatment with XQT ( $n=20$ for each group). The survival rate was monitored every $12 \mathrm{~h}$ for $5 \mathrm{~d}$.

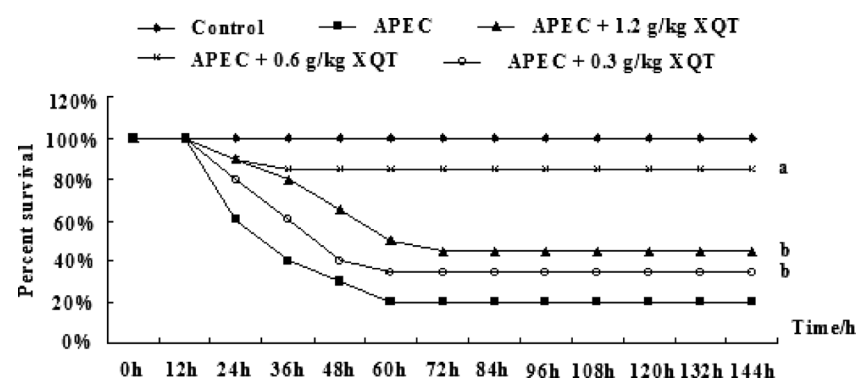

Fig. 2. Effects of Different Dosage of XQT on the Survival Rate of Chickens Challenged with APEC

Chickens were divided into control, APEC and XQT treatment groups $(n=20$ for each group). Chickens in XQT-treated group were given $1.2 \mathrm{~g} / \mathrm{kg}, 0.6 \mathrm{~g} / \mathrm{kg}$ and $0.3 \mathrm{~g} / \mathrm{kg}$ XQT $12 \mathrm{~h}$ before inoculation with $10^{8} \mathrm{CFU}$ of APEC. Chickens in control and APEC groups were given saline. The survival rate was assessed every $12 \mathrm{~h}$ for $6 \mathrm{~d}$ throughout the experiment. ${ }^{\mathrm{a}} p<0.01,{ }^{\mathrm{b}} p>0.05$, compared with the APEC group. 


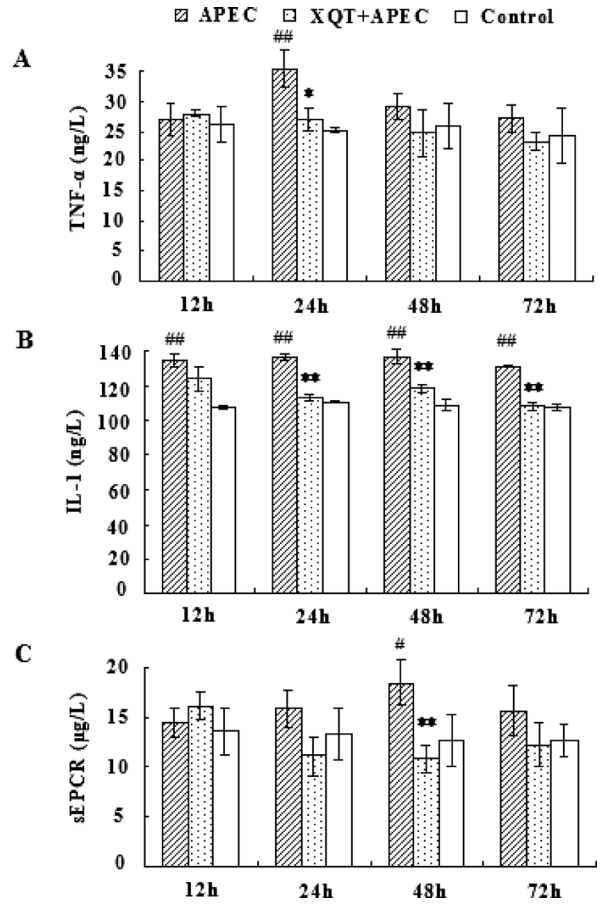

Fig. 3. Influence of XQT on TNF- $\alpha$ (A), IL-1 (B) and sEPCR (C) Induced by APEC

Chickens were given XQT $(0.6 \mathrm{~g} / \mathrm{kg})$ by oral administration $12 \mathrm{~h}$ before challenge with $10^{8} \mathrm{CFU}$ of APEC. Serum levels of TNF- $\alpha$, IL-1, and sEPCR were measured at $12 \mathrm{~h}, 24 \mathrm{~h}, 48 \mathrm{~h}$ and $72 \mathrm{~h}$ following APEC challenge $(n=20)$. Data are presented as mean \pm S.D. of three independent experiments. $* p<0.05, * * p<0.01$, compared with the APEC group. $\# p<0.05$, \#p $<0.01$, compared with the control group.

ner, with the middle dosage group showing $85 \%$ survival $(p<0.01)$, while survival rates in groups treated with 1.2 and $0.3 \mathrm{~g} / \mathrm{kg}$ doses of XQT respectively showed $45 \%$ survival $(p>0.05)$ and $35 \%$ survival $(p>0.05)$.

Effects of XQT on Inflammatory Mediator Concentrations in Chicken Serum To assess the XQT on the production of inflammatory mediators in vivo, the concentrations of TNF- $\alpha$, IL- 1 and sEPCR in the serum were measured at different time points after challenging chickens with APEC. The serum TNF- $\alpha$ levels in all groups are shown in Fig. 3A. The TNF- $\alpha$ concentrations in the APEC group were significantly higher than that in the control group only at $24 \mathrm{~h}$ $(p<0.01)$. At the time point, the TNF- $\alpha$ concentrations in the pretreated XQT group were significantly lower than that in the APEC group $(p<0.05)$.

The serum IL-1 levels in all groups are shown in Fig. 3B. The IL-1 concentrations in the APEC group were significantly higher than those in the control group at $12 \mathrm{~h}, 24 \mathrm{~h}$, $48 \mathrm{~h}$ and $72 \mathrm{~h}(p<0.01)$. As compared with the APEC group, the IL-1 concentrations in the pretreated XQT group were significantly lower $(p<0.01)$ at $24 \mathrm{~h}, 48 \mathrm{~h}$ and $72 \mathrm{~h}$.

The serum sEPCR levels in all groups are shown in Fig. $3 \mathrm{C}$. The sEPCR concentrations in the APEC group were significantly higher than those in the control group at $48 \mathrm{~h}$ $(p<0.05)$. At that time point, the sEPCR concentrations in the pretreated XQT group were significantly lower than those in the APEC group $(p<0.01)$.

\section{DISCUSSION}

The results in this study show that XQT can prevent the mortality of chickens challenged with APEC. This effect was mainly attributed to the middle dosage XQT $(0.6 \mathrm{~g} / \mathrm{kg})$ used to pretreated chickens. It has been shown that APEC proliferates significantly in $6 \mathrm{~h}$ postinoculation, causing acute systemic infection or death. ${ }^{20,21)}$ In our research, the death of infected chickens began from $12 \mathrm{~h}$, and the most benefited doses of XQT decreased the mortality of infected chickens at the death peak during 24 to $48 \mathrm{~h}$ after inoculation of APEC. These data suggest that XQT can prevent the porliferation of APEC and the cascade of events induced by APEC or its toxins in vivo.

Disease states such as local and systemic infection, septic shock, degenerative arthritis and other autoimmune diseases all appear to be mediated, in part, by TNF- $\alpha$ and IL-1. ${ }^{22,23)}$ Blockade of TNF- $\alpha$ and IL- 1 function by TNF- $\alpha$ receptor antagonist and IL-1 receptor antagonist potently inhibits these diseases. ${ }^{24-26)}$ Studies also have shown that endotoxinmediated death can be reversed by manipulation of these cytokine responses in animal models. ${ }^{27}$ ) Thus, we hypothesized that XQT could antagonize the two pro-inflammatory cytokines to prevent the mortality of infected chickens. To attest this assumption, we determined the level of TNF- $\alpha$ and IL-1 in the chickens' plasma. In the absence of XQT, APEC challenge resulted in a burst of IL- 1 and a modest peak in TNF- $\alpha$ concentration, occurring at $12 \mathrm{~h}$ and $24 \mathrm{~h}$, respectively. Serum TNF- $\alpha$ decreased to a normal level after $48 \mathrm{~h}$, while serum IL-1 remained at a high level after $12 \mathrm{~h}$. Pretreatment with the suitable dose of XQT significantly decreased TNF- $\alpha$ and IL-1 concentrations during the death peak of infected chickens, and there was no difference between the XQT pretreated group and the control group. This result suggested that the effect of XQT on cytokine responses is one of the major factors decreasing mortality in this chicken model. We presumed that this effect might be due to the anti-inflammatory activity of components of XQT, because it is reported that Andrographis paniculata ${ }^{28,29)}$ and Astragalus membranaceus ${ }^{30)}$ can inhibit TNF- $\alpha$, IL-1 and other inflammatory mediators through nuclear factor kappa $\mathrm{B}(\mathrm{NF}-\kappa \mathrm{B})$ or other signaling pathways.

Numerous studies have shown that more than $80 \%$ of patients with bacterial infection, especially severe sepsis, had an APC deficiency which is closely associated with mortality in patients. ${ }^{31,32)}$ Gram-negative bacteria and its endotoxin, including inflammatory cytokines TNF- $\alpha$ and IL-1 induced by bacterial infection, led to an evident impairment of the PC system. In our experiment, to investigate the effect of XQT on the PC system, we detected the serum levels of sEPCR, which shed from the endothelial cell surface. EPCR is one of the components of the PC system, ${ }^{33)}$ while binding of its soluble forms (sEPCR) to APC blocks the APC anticoagulant activity, ${ }^{34)}$ which can further amplify inflammation. Gu et $a l^{35)}$ reported that endotoxin could induce an increase of sEPCR in rat serum. In our study, serum sEPCR levels significantly increased at $48 \mathrm{~h}$ after being challenged with APEC alone in the chicken model, and returned toward baseline values at $72 \mathrm{~h}$. Treatment with XQT inhibited the sEPCR increase at $48 \mathrm{~h}$, and the concentrations reached normal levels. According to the analysis above, it is possible that decreasing mortality of XQT may partly be due to restoration of the PC system.

Consider the case of bacterial infection, there is a strong 
association between inflammation and thrombosis. Inflammation can induce local thrombosis, and thrombosis can amplify inflammation. ${ }^{8)}$ Thus, we can regard anti-inflammatory therapies as potentially antithrombotic in the treatment of patients. Many reports have shown that the components of XQT possess anti-inflammatory or antithrombotic activity. Sheeja et al. ${ }^{36)}$ reported that Andrographis paniculata extract could inhibit inflammation induced by carageenan in a mouse model. Some compounds isolated from Andrographis paniculata exhibit an anti-inflammatory property, such as andrographolide $^{37)}$ or andrograpanin. ${ }^{38)}$ Astragalus membranaceus saponins upregulate the PC pathway, exhibiting protection of endothelium or antithrombotic activity. ${ }^{39,40)}$ Rhizoma Cyperi is also extensively used as a Chinese traditional herbal medicine in animal infectious disease therapy. Therefore, XQT protection of infected chickens may be attributed to anti-inflammatory and antithrombotic activity of the components of XQT.

In conclusion, we demonstrated that XQT could increase the survival rate of the chickens challenged with $10^{8} \mathrm{CFU}$ of APEC and decrease the serum levels of TNF- $\alpha$, IL- 1 and sEPCR. The results suggested that XQT had potential antiinflammatory and antithrombotic activity. Thus, XQT may be an effective therapeutic drug for avian colibacillosis, and even other infectious diseases. These findings may contribute to future research not only on the action mechanisms of XQT or the pathogenic mechanisms of APEC, as well as prevention of or therapy for avian colibacillosis, but also on screening of Chinese medicinal active ingredients and new antiinfectious agents.

Acknowledgments This study was supported by the National Key Technology R\&D Program in the People's Republic of China (No. 2008BADB4B04), the National Natural Science Foundation of China (No. 30800834), and the Jilin University Research Fund (to Dr. B. D. Fu).

\section{REFERENCES}

1) Gibbs P. S., Petermann S. R., Wooley R. E., Avian Dis., 48, 751-758 (2004).

2) Dho-Moulin M., Fairbrother J. M., Vet. Res., 30, 299-316 (1999).

3) Seam N., Suffredini A. F., Drug Discov. Today Dis. Mech., 4, 83-93 (2007).

4) Aird W. C., Blood, 101, 3765-3777 (2003).

5) Esmon C. T., Br. J. Haematol., 131, 417-430 (2005).

6) Nelson S., Summer W. R., Infect. Dis. Clin. North Am., 12, 555-567 (1998).

7) Girardin E., Dayer J. M., Schweiz. Med. Wochenschr., 123, 480-491 (1993).

8) Libby P., Simon D. I., Circulation, 103, 1718-1720 (2001).
9) Carvalho A. C., Freeman N. J., J. Crit. Illness, 9, 51-75 (1994).

10) Cao C. Z., Gao Y. M., Li Y., Antalis T. M., Castellino F. J., Zhang L., J. Clin. Invest., 120, 1971-1980 (2010).

11) Esmon C. T., Ann. Med., 34, 598-605 (2002).

12) Fischer D., Nold M. F., Nold-Petry C. A., Furlan A., Veldman A., Vasc. Health Risk Manag., 5, 775-781 (2009).

13) Bae H., Park N., Kim Y., Hong M., Shin M., Kim S.H., Kim J., Cytokine, 51, 259-265 (2010).

14) Mishra U. S., Mishra A., Kumari R., Murthy P. N., Naik B. S., Indian J. Pharm. Sci., 71, 436-438 (2009).

15) Kligler B., Ulbricht C., Basch E., Kirkwood C. D., Abrams T. R., Miranda M., Singh Khalsa K. P., Giles M., Boon H., Woods J., Explore (New York), 2, 25-29 (2006).

16) Li H. B., Ge Y. K., Zhang L., Zheng X. X., Life Sci., 79, 1186-1193 (2006).

17) Cui R., He J., Wang B., Zhang F., Chen G., Yin S., Shen H., Cancer Chemoth. Pharm., 51, 75-80 (2003).

18) Minsook R., Eun H. K., Mison C., J. Ethnopharmacol., 115, 184-193 (2008).

19) Antão E. M., Glodde S., Li G., Sharifi R., Homeier T., Laturnus C., Diehl I., Bethe A., Philipp H. C., Preisinger R., Wieler L. H., Ewers C., Microb. Pathogenesis, 45, 361-369 (2008).

20) Huang H. J., Matsumoto M., Avian Dis., 43, 469-475 (1999).

21) Wooley R. E., Gibbs P. S., Brown T. P., Maurer J. J., Avian Dis., 44, $318-324$ (2000).

22) Dinarello C. A., Int. J. Tissue React., 4, 65-75 (1992).

23) Moldawer L. L., Blood Purificat., 11, 128-133 (1993).

24) Murch S. H., Nutrition, 14, 780-783 (1998).

25) Diel R., Hauer B., Loddenkemper R., Manger B., Krüger K., Z. Rheumatol., 68, 411-416 (2009).

26) Lynn W. A., Cohen J., Clin. Infect. Dis., 20, 143-158 (1995)

27) Ozmen L., Pericin M., Hakimi J., Chizzonite R. A., Wysocka M., Trinchieri G., J. Exp. Med., 180, 907-915 (1994).

28) Chao W. W., Kuo Y. H., Hsieh S. L. Lin B. F., Evid-based Compl. Alt., 9, 1-9 (2009).

29) Chandrasekaran C. V., Gupta A., Agarwal A., J. Ethnopharmacol., 129, 203-207 (2010).

30) Yuan Y., Sun M., Li K. S., World J. Gastroenterol., 15, 3676-3680 (2009).

31) Fisher C. J. Jr., Yan S. B., Crit. Care Med., 28, S49-S56 (2000)

32) Mesters R. M., Helterbrand J., Utterback B. G., Yan B., Chao Y. B., Fernandez J. A., Griffin J. H., Hartman D. L., Crit. Care Med., 28, $2209-2216(2000)$

33) Esmon C. T., Chest, 124, 26S-32S (2003).

34) Regan L. M., Stearns-Kurosawa D. J., Kurosawa S., Mollica J., Fukudome K., Esmon C. T., J. Biol. Chem., 271, 17499-17503 (1996).

35) Gu J. M., Katsuura Y., Ferrell G. L., Grammas P., Esmon C. T., Blood, 95, 1687-1693 (2000).

36) Sheeja K., Shihab P. K., Kuttan G., Immunopharm. Immunother, 28, 129-140 (2006).

37) Parichatikanond W., Suthisisang C., Dhepakson P., Herunsalee A., Int. Immunopharmacol., 10, 1361-1373 (2010).

38) Liu J., Wang Z. T., Ge B. X., Int. Immunopharmacol., 8, 951-958 (2008).

39) Gao X. H., Xu X. X., Pan R., Li Y., Luo Y. B., Xia Y. F., Murata K., Matsuda H., Dai Y., J. Nat. Med., 63, 421-429 (2009).

40) Gao X. H., Xu X. X., Pan R., Wang C., Sheng R., Xia Y. F., Dai Y., Inflammation, in press (2010). 